\title{
Content Destabilization for Head-Mounted Displays
}

\author{
Felix Lauber, Sophia Cook, Andreas Butz \\ HCI Group, University of Munich (LMU), Munich, Germany \\ \{felix.lauber, andreas.butz\}@ifi.lmu.de \\ sophiacook@gmx.de
}

\begin{abstract}
With recent progress in display technology, visual seethrough head-mounted displays are beginning to enter our everyday lives. Especially in cars they may replace head-up displays, as they can theoretically perfectly imitate them but are more flexible to use. However, prior work has shown that both screen- and vehicle-stabilized content suffer from drawbacks such as occlusion or technological limitations. As a potential alternative, we propose three concept alternatives, in which head rotation is used to manipulate the displayed content differently from both of the known stabilization techniques. In a qualitative user study, we identify the best concept proposal and then evaluate it against the established content stabilization techniques. The presented concept is perceived to be more applicable for the proposed use case and effectively reduces some of the known problems of both stabilization techniques.
\end{abstract}

\section{Author Keywords}

Head-mounted display; automotive user interface; head-up display; content stabilization.

\section{ACM Classification Keywords}

H.5.m. Information interfaces and presentation (e.g., HCI): Miscellaneous.

\section{INTRODUCTION}

While head-mounted displays (HMDs) have been used for decades in very specific application domains, they currently seem to have the potential of becoming the next "big thing" of wearable technology in our everyday lives. A promising application area for visual see-through HMDs is in-vehicle interfaces. Head-up displays (HUDs) nowadays are state of the art technology and their advantages, especially in comparison to traditionally used (head-down) displays, already have been proven in many use cases [e.g., 2, 5]. Once the technological limitations of tracking and display technology has been overcome, HMDs could perfectly imitate today's HUDs and even outperform them by the variety of possible use cases: They provide a flexible field

Permission to make digital or hard copies of all or part of this work for personal or classroom use is granted without fee provided that copies are not made or distributed for profit or commercial advantage and that copies bear this notice and the full citation on the first page. Copyrights for components of this work owned by others than ACM must be honored. Abstracting with credit is permitted. To copy otherwise, or republish, to post on servers or to redistribute to lists, requires prior specific permission and/or a fee. Request permissions from Permissions@acm.org.

CHI 2015, April 18 - 23 2015, Seoul, Republic of Korea

Copyright is held by the owner/author(s). Publication rights licensed to ACM.

ACM 978-1-4503-3145-6/15/04 ..\$15.00

http://dx.doi.org/10.1145/2702123.2702161 of view (FOV), they can be seamlessly used inside and outside of vehicles, they are relatively inexpensive and do not need to be integrated into the technical design of the vehicle.

\section{USING HMDS IN CARS}

HMDs have been used in cars in a variety of ways (see [8] for an overview). As the driver's body as a reference system loses importance, one usually differentiates between screen-, vehicle- and world-stabilized content [9]. Concept developers traditionally choose either screen- or vehiclestabilized content to display driving-related data (e.g. speed, speed limits or navigational information). Screen-stabilized content is displayed at a fixed position in relation to the screen (HMD). Thus, there is no need for head-tracking and content can be displayed convincingly even in the small display area of today's HMDs. However, as content is visible independently from the driver's head movements, it may obstruct for example the rear view mirror, traffic signs or even other traffic participants [3]. In contrast, vehiclestabilized content is displayed at a fixed position in relation to the vehicle: if the HMD (respectively the user's head) is moved, content has to be moved accordingly on the HMD. Thus, it strongly depends on the quality of the head tracking and the size of the head-mounted display's field of view. Using today's state of the art tracking systems and HMDs, content is perceived to be relatively unsteady and head movements for as little as a couple of degrees already cause content to (partly) leave the HMD's display area [3]. Besides the technology-based solution to these challenges (such as improving tracking and display technology), we argue that there is also an interface-based approach by moving away from the idea of stabilization (hence the term destabilization). This leads us to a new design space, where head rotation no longer is used to create the illusion of content being displayed at a fixed position in the vehicle, but to provide the best viewing experience in the proposed use case.

\section{BASIC CONCEPT IDEA}

The basic idea is to divide the horizontal FOV of the driver into different zones (Fig. 1). When looking straight ahead, content should be presented as calm and steady as possible. For the concepts in this paper, we chose a position within the HMD's display area that is similar to today's HUD systems (central position in front of the driver, right on top of the dashboard). Given that the driver is looking straight ahead, we thus minimize obstruction of real world objects. There are situations, however, in which the driver has to 
turn his/her head. To circumvent the possibility that the visualization on the HMD would obstruct important information, we track the user's head and change the content appearance so that it is no longer is in his/her primary FOV.

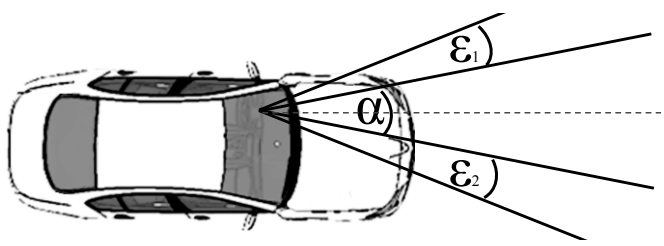

Figure 1: Viewing Zones as defined in our concepts.

\section{GAZE BEHAVIOUR WHILE DRIVING}

To define correct viewing zones, we reviewed typical gaze and head movement behavior while driving. According to a study conducted by Martin et al. [6], the head position of drivers does not exceed a yaw-angle of $18^{\circ}$ to the left and right for $95 \%$ of the time [7]. These values are compliant to basic research in the field of human gaze analysis: especially in situations, where the target position is predictable, a yaw-angle of approx. $15^{\circ}$ to both sides is covered by eye saccades before people turn their head [10]. However, there also are situations in which drivers might need to turn their head further: glancing over one's shoulder while changing lanes, looking into the rear view mirror or gaze changes while performing turning maneuvers [4] are examples. Checking the instrument cluster or interacting with the center stack display might also require head movements away from the street. As a consequence, we defined a $30^{\circ}$ viewing angle ( $\alpha$ in Fig. 1) for situations in which the driver is looking ahead to the street and copes with gaze changes by using eye saccades. Another zone of $10^{\circ}$ to each direction $\left(\varepsilon_{1}\right.$ and $\varepsilon_{2}$ in Fig. 1$)$ is reserved for the content transition of the particular concept.

\section{CONCEPT VARIATIONS}

Traditional content stabilization techniques can be described with functions that translate head-rotation (hr) into rotation values of the virtual (scene-) camera (cr). While in the case of vehicle-stabilized content this function can be denoted as $c r=f(h r)=h r$, it can be denoted as $c r=f(h r)=c$ (constant) for screen-stabilized content. The idea of the following three concept variations is to use translation functions, which are less sensitive to immature tracking technology but at the same time circumvent occlusion problems of screen-stabilized content. To frame the resulting design space, the first two concept approaches use conceptually different (discontinuous respectively exponentially growing) translation functions to manipulate the position of the displayed content. The third concept explores how other display properties (e.g., transparency) can be manipulated by head rotation.

The first concept variation is called Relocate HUD and uses a function which translates head-rotation (hr) into camera rotation (cr) in a discontinuous manner (Fig. 3b). As a result, content is screen-stabilized (zone $\alpha$ ) but changes its structure and position (Fig. 2) according to the driver's head rotation (zones $\varepsilon_{1}$ and $\varepsilon_{2}$ ) until it finally disappears completely (when turning one's head even further).

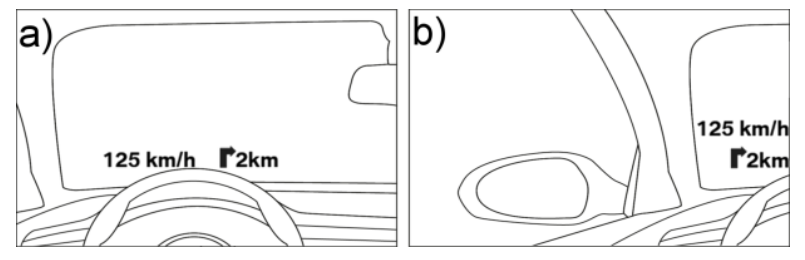

Figure 2: Relocate HUD

The second concept is called Magnetic HUD. Instead of directly (linearly) translating the head rotation into camera rotation, we use an exponentially growing translation function (Fig. 3c). As a result, head movements up to a certain threshold (within zone $\alpha$ ) have only very little impact on the rotation of the (virtual) camera while head movements exceeding this threshold (zones $\varepsilon_{1}$ and $\varepsilon_{2}$ ) cause the virtual content to move very quickly out of vision.
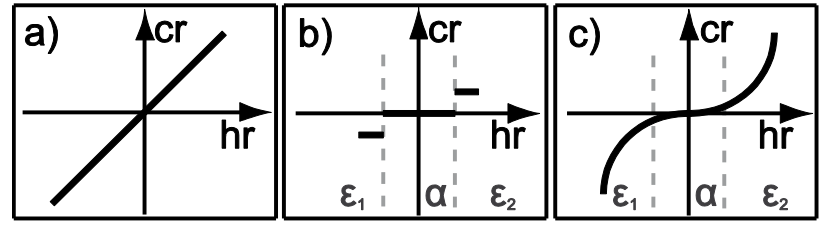

Figure 3: Translation functions of head-rotation (hr) to rotation of the virtual scene camera (cr) for screen-stabilized content (a), the relocate HUD (b) and the magnetic HUD (c)

The last, which we call $\alpha$-blending $H U D$, uses a function that translates increasing head-rotation values linearly into increasing content transparency (Fig. 4). As this is only done within the transition areas $\left(\varepsilon_{1}\right.$ and $\left.\varepsilon_{2}\right)$, content is screen-stabilized when the driver is looking straight ahead (zone $\alpha$ ) and slowly disappears as the head rotation crosses the transition zones $\varepsilon_{1}$ and $\varepsilon_{2}$.

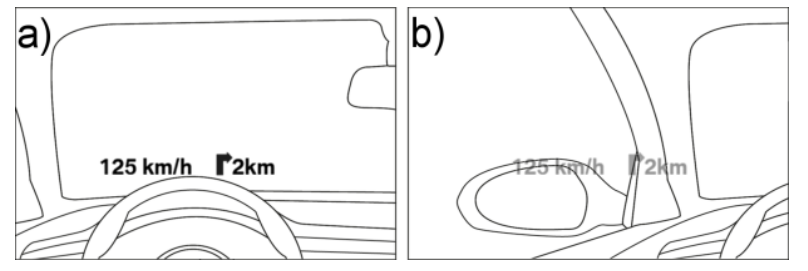

Figure 4: $\alpha$-blending HUD

\section{EVALUATION}

In order to evaluate whether the novel concept variations provide any additional value to HMD-assisted driving, we conducted two studies. In the first study, we assessed which of the three concept variations performs best. In the second study we compared the best performing concept to the baseline concepts vehicle-stabilized and screen-stabilized.

\section{Apparatus, Procedure and Task}

We used the same driving simulation setup for both studies. An industry-grade car mockup was placed approx. 1.5 meters in front of a large, cylindrical $220^{\circ}$ projection 
screen, on which the driving scene was displayed. Three 50 " LCD displays were placed behind the rear view mirrors to display the scene behind the driver. For head tracking we used a state of the art infrared tracking device (ART Smarttrack), which was placed at a distance of approx. 90 $\mathrm{cm}$ at the dashboard centrally in front of the driver. The tracker was used at a frame rate of $60 \mathrm{~Hz}$ in combination with a passive head-mounted GT-5 tracking target and predictive filter algorithms. During the test, subjects wore a binocular optical see-through HMD (LUMUS DK-32). The HMD has a resolution of $1280 \times 720$ px. with a $40^{\circ}$ diagonal field of view. The virtual image is appearing at a distance of $3 \mathrm{~m}$. Brightness was adjusted to $255.4 \mathrm{~cd} / \mathrm{m}^{2}$ with a contrast ratio of 100:1. During driving, the HMD displayed standard HUD content, which is the current driving speed, an optional speed limit information and navigational information.

Subjects were instructed to drive according to the speed limits and follow the instructions of the navigation system. Both studies were conducted in a one factorial (three levels) within-subjects design. After a short test run (approx. 5 min.), in which subjects could get accustomed to the driving simulation and to wearing the HMD, they were asked to complete three test runs. Right before each test run, the instructor calibrated the HMD visualization to ensure equal positioning of the displayed content and instructed the participant in the following test condition. Immediately after each test run we asked participants to fill out a questionnaire, in which subjects were asked to state their level of agreement on a 5-point Likert scale from 0 "not at all" to 5 "total agreement" regarding potential problems of each concept. Besides general aspects of HMD visualizations (such as the restlessness/unsteadiness of the visualization, and the undesired occlusion of real world objects), we also wanted to assess specific parameters of the visual transition (level of annoyance and visual distraction). We used a test course with three track sections: a motorway section followed by a highway section and a section in a big city. We designed 5 tasks, which had to be accomplished in each test run: reading an information of the instrument cluster (1), manipulating the center stack display with the integrated control device (rotary controller in the center stack) (2), merging into a gap while changing lanes (3), an overtaking maneuver (4) and a big crossroads where subjects had to yield to priority traffic (5). In situations 1 and 2 we asked for 3 different chunks of information, to avoid memorizing effects. Conditions were counterbalanced to obviate effects of familiarization. Immediately after each of the situations, subjects were asked to state the level of distraction (on a five-point scale) caused by the concept. After the three test runs had been completed, we asked subjects to fill out a questionnaire, in which they could select their preferred concept and explain their choice.

\section{First Study}

The goal of the first study was to reveal potential differences between the three developed concept variations and to find out which one worked best. In total, 15 subjects (age 22-59, $\mathrm{M}=32.8, \mathrm{SD}=11.9$ ) participated in this study.

\section{Results}

A total of 9 out of the 15 subjects favored the concept $\alpha$ Blending HUD, only 6 voted for Magnetic HUD and no one for Relocate $H U D$. To evaluate the questionnaires and reveal potential differences across conditions, we calculated one factorial repeated measures ANOVAs and Bonferroni corrected post-hoc tests. According to our results, concepts differed significantly from each other regarding the categories distraction, annoyance and restlessness (Fig. 5).

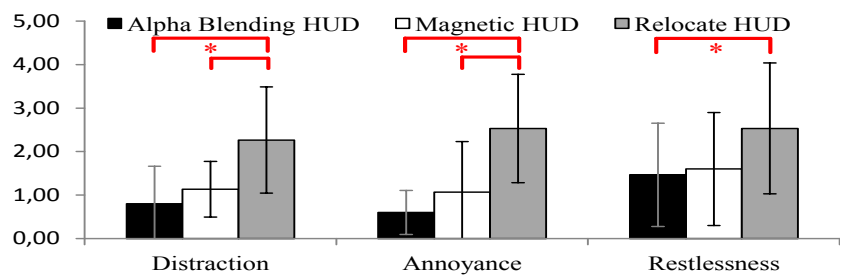

Figure 5: Results of the questionnaires $\left({ }^{*} \mathbf{p}<.05\right)$.

Subjects decided that the relocate $H U D(\mathrm{M}=2.27, \mathrm{SD}=1.22)$ is significantly more distracting than both the magnetic HUD $(\mathrm{M}=1.13, \mathrm{SD}=0.64)$ and the $\alpha$-blending $H U D$ $(\mathrm{M}=0.80, \mathrm{SD}=0.86)$. Analogously relocate HUD $(\mathrm{M}=2.53$, $\mathrm{SD}=1.25$ ) was perceived to be significantly more aggravating than both the magnetic HUD $(\mathrm{M}=1.07$, $\mathrm{SD}=1.16)$ and the $\alpha$-blending $H U D(\mathrm{M}=0.60, \mathrm{SD}=0.51)$. Subjects also found the $\alpha$-blending $H U D$ visualization $(\mathrm{M}=1.47, \mathrm{SD}=1.19)$ significantly less restless than the relocate HUD $(\mathrm{M}=2.53, \mathrm{SD}=1.51)$. Additionally 5 subjects mentioned that they were irritated by the "jerky movements" of the relocate HUD. In contrast, 5 subjects stated, that they liked the seamless transition of the $\alpha$ blending HUD. Regarding the traffic situations, the only significant difference occurred in situation 3 (merging into the gap). Analog to the other qualitative data, $\alpha$-blending $H U D$ was perceived to be significantly less disturbing $(\mathrm{M}=0.57, \quad \mathrm{SD}=0.93)$, than relocate $H U D \quad(\mathrm{M}=1.71$, $\mathrm{SD}=1.26$ ), while magnetic $H U D$ was between the two $(\mathrm{M}=1.07, \mathrm{SD}=1.26)$.

\section{Baseline Study}

In the first study, $\alpha$-Blending $H U D$ outperformed the other two concept variations. In a next step, we compared $\alpha$ Blending HUD to the established stabilization techniques vehicle-stabilized and screen-stabilized. We used the same study design as in the first evaluation. A total of 24 (different) subjects participated in the second study (age 22$50, \mathrm{M}=27.7, \mathrm{SD}=7.5$ ).

\section{Results}

A total of 16 subjects favored the concept $\alpha$-Blending $H U D$, over vehicle-stabilized (5 subjects) and screen-stabilized (3 subjects) content. We used the same questionnaires as in the first study to learn more about the reason for the subject's choice. According to our results, the amount of occlusion (inside and outside the vehicle), the overall unsteadiness of 
the visualization and the quality of the interplay between the visualization and the driving task were the crucial factors in this comparison (Fig. 6).

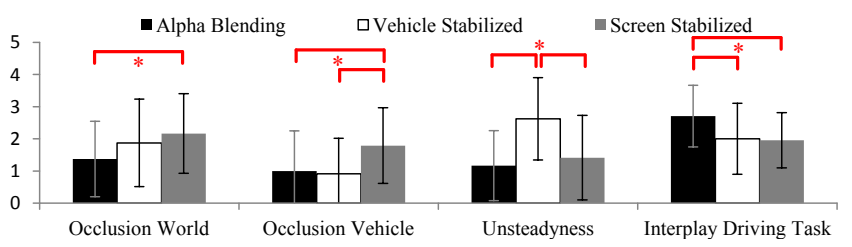

Figure 6: Results of the questionnaires $(p<.05)$.

Subjects claimed that real world objects were significantly less occluded with $\alpha$-Blending HUD $(\mathrm{M}=1.38, \mathrm{SD}=1.17)$ than with screen-stabilized content $(\mathrm{M}=2.17, \mathrm{SD}=1.24)$ while there was no significant difference to vehiclestabilized content $(\mathrm{M}=1.88, \mathrm{SD}=1.36)$. Occlusion problems with parts of the vehicle (e.g., in-vehicle displays) were significantly more likely with screen-stabilized content $(\mathrm{M}=1.79, \mathrm{SD}=1.18)$ than with both, $\alpha$-Blending HUD $(\mathrm{M}=1.00, \quad \mathrm{SD}=1.25)$ and vehicle-stabilized content $(\mathrm{M}=0.92, \mathrm{SD}=1.10)$. The problem of perceiving content as unsteady was significantly bigger with vehicle-stabilized content $(\mathrm{M}=2.63, \mathrm{SD}=1.28)$ than with $\alpha$-Blending $H U D$ $(\mathrm{M}=1.17, \mathrm{SD}=1.09)$ and screen-stabilized content $(\mathrm{M}=1.42$, $\mathrm{SD}=1.32)$. All in all, subjects felt that $\alpha$-Blending $H U D$ $(\mathrm{M}=2.71, \mathrm{SD}=0.95)$ was significantly more suitable to the driving task than screen-stabilized $(\mathrm{M}=1.96, \mathrm{SD}=0.86)$ content and vehicle-stabilized content $(\mathrm{M}=2.00, \mathrm{SD}=1.10)$.

Regarding the specific driving situations, subjects rated the $\alpha$-Blending HUD $(\mathrm{M}=0.70, \mathrm{SD}=0.69)$ significantly less disturbing than vehicle-stabilized $(\mathrm{M}=1.58, \mathrm{SD}=1.18)$ and screen-stabilized content $(\mathrm{M}=1.25, \quad \mathrm{SD}=0.85)$ when changing lanes. When manipulating the center stack display, $\alpha$-Blending HUD $(\mathrm{M}=0.71, \mathrm{SD}=0.81)$ and vehiclestabilized content $(\mathrm{M}=0.67, \mathrm{SD}=0.70)$ was significantly less disturbing than screen-stabilized content $(M=1.29$, $\mathrm{SD}=0.91)$.

\section{CONCLUSION}

In this paper we proposed three concept variations, which circumvent known problems for vehicle-stabilized and screen-stabilized content in HMDs. Instead of translating head movement linearly into movements of the virtual camera (vehicle-stabilization), we explore potential benefits of destabilizing content by using a non-linear and a discontinuous function. Furthermore, instead of discarding head-rotation altogether (screen-stabilization), we explore how it might influence other characteristics of a user interface such as structural appearance and translucency.

In a first user study, we identified $\alpha$-Blending $H U D$ as the best concept variation. While the relocate $H U D$ was perceived to be very distracting because of the moving elements within the peripheral FOV, the fading effect of $\alpha$ Blending HUD was perceived to be the most pleasant and suitable for the driving task. In our second study, we compared $\alpha$-Blending HUD with screen-stabilized and vehicle-stabilized content. As expected, it systematically outperformed vehicle-stabilized content with regard to its steady and discreet appearance, while it had significant advantages to screen-stabilized content with regard to nonexisting occlusion effects. All in all, $\alpha$-Blending $H U D$ was rated significantly better for the task of driving a car than the two eligible existing stabilization techniques.

While head rotation traditionally is used to mimic a fixed content position in an external frame of reference, the proposed concepts open a new design space for interface development for HMDs. In the future, it will be desirable to further examine this design space and to combine it with other contextual cues to alter content appearance in an HMD. The car's current speed, for example, might additionally contribute to predict how content should be presented in order to avoid any kind of driver distraction.

\section{REFERENCES}

1. Doshi, A. and Trivedi, M. M. Head and eye gaze dynamics during visual attention shifts in complex environments. Journal of vision, 12(2), 1-16, 2012.

2. Kiefer, R. Defining the "HUD benefit time window". Vision in Vehicles-VI, 133-142. 1998.

3. Lauber, F., and Butz, A. Are HMDs the better HUDs? In Proc. ISMAR, 267-268, IEEE 2013.

4. Liebner, M., Ruhhammer, C., Klanner, F., Stiller, C. Generic driver intent inference based on parametric models. In Proc. ITSC, 268-275, IEEE 2013.

5. Liu, Y.-C. and Wen, M.-H. Comparison of head-up display (HUD) vs. head-down display (HDD): driving performance of commercial vehicle operators in Taiwan. International Journal of Human-Computer Studies, 679-697. 2004.

6. Martin, S., Tawari A., Chutorian, E.-M., Cheng, S. Y., and Trivedi, M. M. On the design and evaluation of robust head pose for visual user interfaces: Algorithms, databases, and comparisons. In Proc. AutomotiveUI 2012, 149-154.

7. Martin, S., Tawari, A. and Trivedi, M.M. Monitoring head dynamics for driver assistance systems: A multiperspective approach. In Proc. ITSC, 2286 -2291. IEEE 2009.

8. Plavšic, M., Duschl, M., Tönnis, M., Bubb, H. and Klinker, G. Ergonomic Design and Evaluation of Augmented Reality Based Cautionary Warnings for Driving Assistance in Urban Environments. In Proc. International Ergonomics Association. 2009.

9. Wickens, C. D., Vincow, M., and Yeh, M. Design applications of visual spatial thinking: The importance of frame of reference. The Cambridge handbook of visuospatial thinking. 383-425. 2005.

10.Zangemeister, W. H. and Stark, L. Types of gaze movement: variable interactions of eye and head movements. Experimental neurology, 77(3), 563-577. 1982. 